PROCEEDINGS OF THE

AMERICAN MATHEMATICAL SOCIETY

Volume 140, Number 10, October 2012, Pages 3333-3348

S 0002-9939(2011)11167-5

Article electronically published on October 6, 2011

\title{
ON ISOMORPHISM PROBLEMS FOR VERTEX OPERATOR ALGEBRAS ASSOCIATED WITH EVEN LATTICES
}

\author{
HIROKI SHIMAKURA
}

(Communicated by Gail R. Letzter)

\begin{abstract}
AвSTRACT. In this article, we completely determine the isomorphism classes of lattice vertex operator algebras and the vertex operator subalgebras fixed by a lift of the -1-isometry of the lattice. We also provide similar results for certain even lattices associated with doubly-even binary codes.
\end{abstract}

\section{INTRODUCTION}

The lattice vertex operator algebra (VOA) $V_{L}$ associated with an even lattice $L$ is a fundamental example in VOA theory ([Bo86, FLM88] $)$. The VOA $V_{L}$ has an automorphism of order 2 lifted from the -1-isometry of $L$, and the fixed-point subspace $V_{L}^{+}$is a subVOA of $V_{L}$. The famous moonshine VOA $V^{\natural}$ was constructed in [FLM88] as an extension of $V_{\Lambda}^{+}$associated with the Leech lattice $\Lambda$. Therefore, if $L$ is a sublattice of $\Lambda$, then the VOA $V_{L}^{+}$is contained in $V^{\natural}$. Many group-theoretical properties of the automorphism group of $V^{\natural}$, the Monster simple group, can be studied using the subVOA $V_{L}^{+}$. For instance, certain maximal 2-local subgroups of the Monster simple group were described using $V_{L}^{+}$([Sh07, Sh11]).

It is well known that the VOAs $V_{L}$ and $V_{N}$ are isomorphic if and only if $L$ and $N$ are isomorphic. However it was shown in [Sh06] that the VOAs $V_{D_{16}^{+}}^{+}$and $V_{E_{8}^{2}}^{+}$ are isomorphic, where $D_{16}^{+}$and $E_{8}^{2}$ are (nonisomorphic) even unimodular lattices of rank 16. Hence it is natural to ask if this case is the only exceptional case.

Given a doubly-even binary code $C$, one can obtain an even lattice $\mathcal{L}(C)=$ $\frac{1}{\sqrt{2}} \rho^{-1}(C)$ and a certain sublattice $\mathcal{L}^{+}(C)$ of index 2 , where $\rho$ is the canonical map from $\mathbb{Z}^{n}$ to $\mathbb{Z}_{2}^{n}$. The Leech lattice $\Lambda$ can be constructed as an overlattice of $\mathcal{L}^{+}\left(G_{24}\right)$ (CS99]), where $G_{24}$ is the extended binary Golay code. The construction of $V^{\natural}$ in FLM88 is, in some sense, analogous to this construction. Since there are many analogies among binary codes, lattices and VOAs ([Hö95, Hö03a, Hö08, Sh11]), it is natural to ask if the VOAs $V_{L}$ and $V_{L}^{+}$would behave like $\mathcal{L}(C)$ and $\mathcal{L}^{+}(C)$, respectively.

Received by the editors October 25, 2009 and, in revised form, May 4, 2010; January 18, 2011; and April 4, 2011.

2010 Mathematics Subject Classification. Primary 17B69; Secondary 11H06, 11H71.

The author was partially supported by Grants-in-Aid for Scientific Research (No. 20549004) and Excellent Young Researcher Overseas Visit Program, JSPS.

(C) 2011 American Mathematical Society
public domain 28 years from publication

Reverts to public domain 28 years from publication 
TABLE 1. Isomorphism problems and the answers

\begin{tabular}{|c|c|}
\hline Problem & Answer \\
\hline \hline $\mathcal{L}(C) \cong \mathcal{L}(D)$ & $C \cong D$ \\
\hline$V_{L} \cong V_{N}$ & $L \cong N$ \\
\hline \hline $\mathcal{L}^{+}(C) \cong \mathcal{L}^{+}(D)$ & $C \cong D$ or $\{C, D\}=\left\{e_{8}^{2}, d_{16}^{+}\right\}$ \\
\hline$V_{L}^{+} \cong V_{N}^{+}$ & $L \cong N$ or $\{L, N\}=\left\{E_{8}^{2}, D_{16}^{+}\right\}$ \\
\hline \hline $\mathcal{L}^{+}(C) \cong \mathcal{L}(D)$ & $C \cong \mathcal{C}(K)$ and $D \cong \mathcal{C}^{+}(K)$ \\
\hline$V_{L}^{+} \cong V_{N}$ & $L \cong \mathcal{L}(C)$ and $N \cong \mathcal{L}^{+}(C)$ \\
\hline
\end{tabular}

In this article, we completely determine when $V_{L}^{+}$and $V_{N}^{+}$are isomorphic as well as when $V_{L}^{+}$and $V_{N}$ are isomorphic. We also obtain similar results for the even lattices $\mathcal{L}(C)$ and $\mathcal{L}^{+}(C)$ associated with doubly-even binary codes $C$. As an observation, we notice that the isomorphism types of the VOAs $V_{L}$ and $V_{L}^{+}$are closely related to those of the lattices $\mathcal{L}(C)$ and $\mathcal{L}^{+}(C)$ (see Table 1). Note that $\mathcal{C}(K)$ and $\mathcal{C}^{+}(K)$ in the table are binary codes obtained from Kleinian codes $K$ ([Hö03a] ) and that some results for the even lattices were already given in KKM91.

Next, let us explain our method for the case $V_{L}^{+} \cong V_{N}^{+}$(Theorem 3.9). We note that $V_{L} \cong V_{N}$ if and only if $L \cong N$ (cf. Proposition [3.1), and the case $V_{L}^{+} \cong V_{N}$ (Theorem 3.10) can be solved easily by Theorem 3.9. Clearly, $L \cong N$ implies $V_{L}^{+} \cong V_{N}^{+}$, and it was shown in [Sh06] that $V_{E_{8}^{2}}^{+} \cong V_{D_{16}^{+}}^{+}$. We assume that $V_{L}^{+} \cong V_{N}^{+}$as VOAs. Let us consider the irreducible simple current module $V_{N}^{-}$. By the assumption, we regard the irreducible $V_{N}^{+}$-module $V_{N}^{-}$as an irreducible $V_{L}^{+}$-module $M$. The key is that the properties of $M$ are similar to those of $V_{N}^{-}$. By the classification of the irreducible modules for $V_{L}^{+}$([DN99, AD04]), we have $M \cong V_{L}^{-}, V_{\lambda+L}^{\varepsilon}(\lambda \notin L, \varepsilon \in\{ \pm\})$ or $V_{L}^{T_{\chi}, \pm}$ as $V_{L}^{+}$-modules.

(i) If $M \cong V_{L}^{-}$, then $V_{N} \cong V_{N}^{+} \oplus V_{N}^{-} \cong V_{L}^{+} \oplus V_{L}^{-} \cong V_{L}$ as VOAs and $L \cong N$.

(ii) Assume $M \cong V_{\lambda+L}^{\varepsilon}$. We may assume that $\varepsilon=+$ up to conjugation ([Sh04]). Since the characters of $V_{L}^{-}$and $V_{\lambda+L}^{+}$are the same, we obtain an equation about the numbers of norm 2 vectors in $L$ and $\lambda+L$. By the characterization of $\mathcal{L}^{+}(C)$ in [Sh06, $L \cong \mathcal{L}^{+}(C)$ and $L+\mathbb{Z} \lambda \cong \mathcal{L}(C)$ for some doubly-even binary code $C$. Moreover, since $V_{\mathcal{L}(C)}^{+} \cong V_{\mathcal{L}^{+}(C)}($ [FLM88] $)$, we obtain $V_{L} \cong V_{L}^{+} \oplus V_{L}^{-} \cong$ $V_{L}^{+} \oplus V_{\lambda+L}^{+} \cong V_{N}$ as VOAs, and hence $L \cong N$.

(iii) Assume $M \cong V_{L}^{T_{\chi},+}$. Since the lowest weights are the same, the rank of $L$ is 8 . Since $M$ is a self-dual simple current, $\sqrt{2} L^{*}$ is even. Hence $L$ contains a sublattice isomorphic to $\sqrt{2} E_{8}$. By the same arguments for $N, N$ has the same property. Since the discriminant groups of $L$ and $N$ are isomorphic, we have $L \cong N$.

(iv) Assume $M \cong V_{L}^{T_{\chi},-}$. Then the rank of $L$ is 16 . Moreover, both $L$ and $N$ are unimodular, or $L \cong \mathcal{L}^{+}(C), N \cong \mathcal{L}^{+}(D)$ for some doubly-even binary codes $C$ and $D$ with the same weight enumerator. The first case is an exceptional case. In the latter case, either both $C$ and $D$ are self-dual, or $C \cong \mathcal{C}^{+}(K), D \cong \mathcal{C}^{+}(J)$ for some Kleinian codes $K$ and $J$ with the same weight enumerator. If both $C$ and $D$ are self-dual, then $L \cong \mathcal{L}^{+}(C) \cong \mathcal{L}^{+}(D) \cong N$. By the classification of even Kleinian codes of small length ( 
This article also contains proofs for the corresponding results in lattices. These should be useful for understanding the arguments in VOAs, since the approach in lattices is quite similar to that in VOAs.

\section{Preliminaries}

In this section, we recall or give some definitions and facts required in this article.

1.1. Kleinian codes. In this subsection, we recall the basic definitions for Kleinian codes (cf. [Hö03a]).

Denote the elements of the Kleinian four group $\mathbb{K} \cong \mathbb{Z}_{2} \times \mathbb{Z}_{2}$ by $0, a, b, c$, where 0 is the identity. A (linear) Kleinian code $K$ of length $n$ is a subgroup of $\mathbb{K}^{n} \cong \mathbb{Z}_{2}^{2 n}$. The dimension of $K$ is $t$ if $K$ has $4^{t}$ elements, where $t \in \frac{1}{2} \mathbb{Z}$. The weight $\operatorname{wt}(x)$ of $x=\left(x_{i}\right) \in \mathbb{K}^{n}$ is the number of nonzero $x_{i}$. For $m \in \mathbb{Z}$, let $K(m)$ denote the set of all elements of weight $m$ in $K$. A Kleinian code $K$ is even if $\operatorname{wt}(k) \in 2 \mathbb{Z}$ for all $k \in K$. The symmetric bilinear product $\mathbb{K}^{n} \times \mathbb{K}^{n} \rightarrow \mathbb{F}_{2}$ is defined by $x \cdot y=\sum_{i=1}^{n} x_{i} \cdot y_{i}$, where $a \cdot b=b \cdot a=1, a \cdot c=c \cdot a=1, c \cdot b=b \cdot c=1$, and zero otherwise. A Kleinian code is self-dual if it is equal to its orthogonal complement. Two Kleinian codes are equivalent if one of them is obtained from the other by a permutation of the coordinates together with a permutation of the symbols $a, b$ and $c$ at each coordinate. The weight enumerator of $K$ is defined by

$$
W_{K}(X, Y)=\sum_{k \in K} X^{\mathrm{wt}(k)} Y^{n-\mathrm{wt}(k)} .
$$

Up to length 8, self-dual Kleinian codes were classified in Hö03a.

Lemma 1.1 ([Нӧ03a $)$.

(1) There is exactly one even self-dual Kleinian code of length 2, up to equivalence. It is equivalent to $\epsilon_{2}$ generated by $(a a)$ and $(b b)$.

(2) There are exactly two even self-dual Kleinian codes of length 4, up to equivalence. They are equivalent to $\epsilon_{2}^{2}$ and $\delta_{4}^{+}$, where $\delta_{4}^{+}$is generated by $(a a 00),(a 0 a 0),(a 00 a)$, and $(b b b b)$.

1.2. Binary codes. In this subsection, we recall the basic definitions for binary codes (cf. CS99).

A binary (linear) code of length $n$ is a subspace of $\mathbb{F}_{2}^{n}$. The weight wt $(x)$ of $x=$ $\left(x_{i}\right) \in \mathbb{F}_{2}^{n}$ is the number of nonzero $x_{i}$. For a subset $D$ of $\mathbb{F}_{2}^{n}$, we denote the set of all elements in $D$ of weight $m$ by $D(m)$. A binary code $C$ is doubly-even if wt $(x) \in 4 \mathbb{Z}$ for all $x \in C$. The dual code $C^{\perp}$ of $C$ is defined by $C^{\perp}=\left\{x \in \mathbb{F}_{2}^{n} \mid\langle x, C\rangle=0\right\}$, where $\langle x, y\rangle=\sum_{i=1}^{n} x_{i} y_{i}$. A binary code $C$ is self-dual if $C=C^{\perp}$. Two binary codes are equivalent if one of them is obtained from the other by a permutation of the coordinates. The weight enumerator of a coset $x+C \in C^{\perp} / C$ is defined by

$$
W_{x+C}(X, Y)=\sum_{y \in x+C} X^{\mathrm{wt}(y)} Y^{n-\mathrm{wt}(y)}=\sum_{m=0}^{n}|(x+C)(m)| X^{m} Y^{n-m} .
$$

We refer to [CS99] for the details about the binary codes $e_{8}$ and $d_{16}^{+}$.

Lemma 1.2 (cf. [CS99]).

(1) The extended Hamming code $e_{8}$ is the unique doubly-even self-dual code of length 8 , up to equivalence. 
(2) There are exactly two doubly-even self-dual codes of length 16, up to equivalence. They are equivalent to $e_{8}^{2}$ and $d_{16}^{+}$.

1.3. Lattices. In this subsection, we recall the basic definitions for lattices (cf. CS99]).

Let $(\cdot, \cdot)$ be a positive-definite symmetric bilinear form on $\mathbb{R}^{n}$. The norm of $v \in \mathbb{R}^{n}$ is $(v, v)$. For a subset $U \subset \mathbb{R}^{n}$, let us denote by $U(m)$ the set of all vectors of norm $m$ in $U$. A subset $L$ of $\mathbb{R}^{n}$ is a (positive-definite) lattice of rank $n$ if there is a basis $e_{1}, e_{2}, \ldots, e_{n}$ of $\mathbb{R}^{n}$ satisfying $L=\bigoplus_{i=1}^{n} \mathbb{Z} e_{i}$. The dual lattice $L^{*}$ of $L$ is defined by $L^{*}=\left\{u \in \mathbb{R}^{n} \mid(u, L) \subset \mathbb{Z}\right\}$. The discriminant group of $L$ is the quotient group $L^{*} / L$. A lattice $L$ is even if $(v, v) \in 2 \mathbb{Z}$ for all $v \in L$, and $L$ is unimodular if $L=L^{*}$. Two lattices are isomorphic if one of them is obtained from the other by an orthogonal transformation of $\mathbb{R}^{n}$. The theta series of a coset $\lambda+L \in L^{*} / L$ is defined by

$$
\Theta_{\lambda+L}(q)=\sum_{v \in \lambda+L} q^{(v, v) / 2}=\sum_{m=0}^{\infty}|(\lambda+L)(m)| q^{m / 2} .
$$

We refer to CS99] for the details about the lattices $E_{8}$ and $D_{16}^{+}$.

Lemma 1.3 (cf. [Wi41, CS99]).

(1) The root lattice $E_{8}$ is the unique even unimodular lattice of rank 8, up to isomorphism.

(2) There are exactly two even unimodular lattices of rank 16, up to isomorphism. They are isomorphic to $E_{8}^{2}$ and $D_{16}^{+}$.

1.4. Vertex operator algebras. In this subsection, we recall the basic definitions for vertex operator algebras (cf. [Bo86, FLM88, FHL93]). Throughout this article, all VOAs are defined over the field $\mathbb{C}$ of complex numbers.

A vertex operator algebra $(\mathrm{VOA}) V$ is a $\mathbb{Z}_{\geq 0}$-graded vector space $V=\bigoplus_{m \in \mathbb{Z}>0} V_{m}$ that is equipped with a linear map $Y: V \rightarrow(\operatorname{End}(V))\left[\left[z, z^{-1}\right]\right], v \mapsto Y(v, z)=$ $\sum_{i \in \mathbb{Z}} a_{i} z^{-i-1}$ and nonzero vectors $\mathbf{1}_{V}$ and $\omega_{V}$ satisfying a number of conditions ([Bo86, FLM88]). We often denote it by $(V, Y)$ or $V$. Two VOAs $(V, Y)$ and $\left(V^{\prime}, Y^{\prime}\right)$ are said to be isomorphic if there exists a linear isomorphism $g$ from $V$ to $V^{\prime}$ such that $g Y(v, z) w=Y^{\prime}(g v, z) g w$ for all $v, w \in V$ and $g\left(\omega_{V}\right)=\omega_{V^{\prime}}$.

An (ordinary) module $\left(M, Y_{M}\right)$ for a VOA $V$ is a $\mathbb{C}$-graded vector space $M=$ $\bigoplus_{m \in \mathbb{C}} M_{m}$ equipped with a linear map $Y_{M}: V \rightarrow(\operatorname{End}(M))\left[\left[z, z^{-1}\right]\right]$ satisfying a number of conditions (FHL93]). We often denote it by $M$ and its isomorphism class by $[M]$. The weight of a homogeneous vector $v \in M_{k}$ is $k$. The character of $M$ is defined by

$$
\operatorname{ch}_{M}(q)=q^{-n / 24} \sum_{m \in \mathbb{C}} \operatorname{dim} M_{m} q^{m}
$$

where $n$ is the central charge of $V$. A module is self-dual if its contragredient module ([FHL93]) is isomorphic to itself. If $M$ is irreducible, then there exists $h \in \mathbb{C}$ such that $M=\bigoplus_{m \in \mathbb{Z}_{\geq 0}} M_{h+m}$ and $M_{h} \neq 0$. The number $h$ is called the lowest weight of $M$.

A VOA $V$ is of CFT type if $V_{0}=\mathbb{C} 1$, and $V$ is $C_{2}$-cofinite if $V / \operatorname{Span}_{\mathbb{C}}\left\{a_{-2} b \mid\right.$ $a, b \in V\}$ is finite-dimensional. A VOA $V$ is simple if it is an irreducible $V$-module, and $V$ is rational if any module is completely reducible. A rational simple VOA $V$ is holomorphic if any irreducible module is isomorphic to $V$.

The following was proved in DM04b. For the lattice VOA $V_{L}$, see Section 1.5. 
Lemma 1.4 (DM04b) $)$.

(1) The lattice VOA $V_{E_{8}}$ is the unique $C_{2}$-cofinite holomorphic VOA of CFT type of central charge 8 up to isomorphism.

(2) There are exactly two $C_{2}$-cofinite holomorphic VOAs of CFT type of central charge 16 up to isomorphism. They are isomorphic to $V_{E_{8}^{2}}$ and $V_{D_{16}^{+}}$.

Let $M_{a}, M_{b}, M_{c}$ be modules for a simple VOA $V$. Let $N_{M_{a}, M_{b}}^{M_{c}}$ denote the dimension of the space of all intertwining operators of type $M_{a} \times M_{b} \rightarrow M_{c}$, which is called the fusion rule ([FHL93]). By the definition of the fusion rules, $N_{M_{a}, M_{b}}^{M_{c}}=N_{N_{a}, N_{b}}^{N_{c}}$ if $M_{x} \cong N_{x}$ as $V$-modules for all $x=a, b, c$. Hence, the fusion rules are determined by the isomorphism classes of $V$-modules. Let $R(V)$ denote the set of all isomorphism classes of irreducible $V$-modules. For convenience, we use the following expression:

$$
\left[M_{a}\right] \times\left[M_{b}\right]=\sum_{[M] \in R(V)} N_{M_{a}, M_{b}}^{M}[M]
$$

which is also called the fusion rule.

Let $V(0)$ be a simple VOA. An irreducible $V(0)$-module $M^{1}$ is called a simple current if for any irreducible $V(0)$-module $M^{2}$, there exists a unique irreducible $V(0)$-module $M^{3}$ such that $\left[M^{1}\right] \times\left[M^{2}\right]=\left[M^{3}\right]$. A simple VOA $V$ is called a simple current extension of $V(0)$ graded by a finite abelian group $A$ if $V$ is the direct sum of nonisomorphic irreducible simple current $V(0)$-modules $\{V(\alpha) \mid \alpha \in A\}$ and for all $\alpha, \beta \in A$, the fusion rule $[V(\alpha)] \times[V(\beta)]=[V(\alpha+\beta)]$ holds. The uniqueness of simple current extensions was established as follows.

Proposition 1.5 ([DM04, Proposition 4.3] (cf. [Hö03b, Theorem 4.3])). Let (V,Y) be a simple current extension of a simple VOA V(0). Then the VOA structure of $V$ as a simple current extension of $V(0)$ is uniquely determined by the $V(0)$-module structure of $V$; that is, if $(\tilde{V}, \tilde{Y})$ has a VOA structure with $V=\tilde{V}$ as vector spaces and $Y(v, z)=\tilde{Y}(v, z)$ for all $v \in V(0)$, then the $\operatorname{VOAs}(V, Y)$ and $(\tilde{V}, \tilde{Y})$ are isomorphic.

When every irreducible $V$-module is a simple current, $\times$ is a binary operation on $R(V)$. In addition, if $\times$ satisfies the associative law, then $(R(V), \times)$ is a group, which we call the fusion group of $V$.

1.5. Constructions of binary codes, lattices and VOAs. First, let us consider the binary codes $\mathcal{C}(K)$ and $\mathcal{C}^{+}(K)$ of length $4 m$ obtained from a Kleinian code $K$ of length $m$ [Hö03a, Section 7].

Construction A:

$$
\mathcal{C}(K)=\hat{K}+d_{4}^{m},
$$

where ${ }^{\wedge}: \mathbb{K}^{m} \rightarrow \mathbb{F}_{2}^{4 m}$ is the map induced from $\mathbb{K} \rightarrow \mathbb{F}_{2}^{4}, 0 \mapsto(0000), a \mapsto(1100), b \mapsto$ $(1010), c \mapsto(0110)$, and $d_{4}^{m}=\{(0000),(1111)\}^{m}$.

Construction B:

$$
\mathcal{C}^{+}(K)=\hat{K}+\left(d_{4}^{m}\right)_{0},
$$

where ${ }^{\wedge}: \mathbb{K}^{m} \rightarrow \mathbb{F}_{2}^{4 m}$ is defined as before and $\left(d_{4}^{m}\right)_{0}$ is the subcode of $d_{4}^{n}$ consisting of vectors of weight divisible by 8 .

Lemma 1.6 (cf. [Hö03a, Lemmas 2 and 3]). Let $K$ be a Kleinian code of length $m$. 
(1) If $K$ is self-dual, then so is $\mathcal{C}(K)$.

(2) If $K$ is even, then both $\mathcal{C}(K)$ and $\mathcal{C}^{+}(K)$ are doubly-even.

(3) The weight enumerators of $\mathcal{C}(K)$ and $\mathcal{C}^{+}(K)$ are given as follows:

$$
\begin{aligned}
W_{\mathcal{C}(K)}(X, Y) & =W_{K}\left(X^{4}+Y^{4}, 2 X^{2} Y^{2}\right), \\
W_{\mathcal{C}^{+}(K)}(X, Y) & =\frac{1}{2}\left(W_{K}\left(X^{4}+Y^{4}, 2 X^{2} Y^{2}\right)+\left(X^{4}-Y^{4}\right)^{m}\right) .
\end{aligned}
$$

Let us consider some examples.

\section{Lemma 1.7.}

(1) The binary code $\mathcal{C}\left(\epsilon_{2}\right)$ is equivalent to $e_{8}$.

(2) The binary codes $\mathcal{C}\left(\epsilon_{2}^{2}\right)$ and $\mathcal{C}\left(\delta_{4}^{+}\right)$are equivalent to $e_{8}^{2}$ and $d_{16}^{+}$, respectively.

(3) The binary codes $\mathcal{C}^{+}\left(\epsilon_{2}^{2}\right)$ and $\mathcal{C}^{+}\left(\delta_{4}^{+}\right)$are equivalent.

Next, let us consider the lattices $\mathcal{L}(C)$ and $\mathcal{L}^{+}(C)$ of rank $n$ obtained from a binary code $C$ of length $n$ ([CS99]). Let $\alpha_{1}, \alpha_{2}, \ldots, \alpha_{n}$ be an orthogonal basis of $\mathbb{R}^{n}$ of norm 2 .

Construction A:

$$
\mathcal{L}(C)=\sum_{i=1}^{n} \mathbb{Z} \alpha_{i}+\sum_{c \in C} \mathbb{Z} \frac{1}{2} \alpha_{c}
$$

where $\alpha_{c}=\sum_{i=1}^{n} c_{i} \alpha_{i}$ and $c_{i} \in\{0,1\}$.

Construction B:

$$
\mathcal{L}^{+}(C)=\sum_{1 \leq i, j \leq n} \mathbb{Z}\left(\alpha_{i}+\alpha_{j}\right)+\sum_{c \in C} \mathbb{Z} \frac{1}{2} \alpha_{c},
$$

where $\alpha_{c}$ is defined as before.

Lemma 1.8 (CS99). Let $C$ be a binary code of length $n$.

(1) If $C$ is self-dual, then $\mathcal{L}(C)$ is unimodular.

(2) If $C$ is doubly-even, then both $\mathcal{L}(C)$ and $\mathcal{L}^{+}(C)$ are even.

(3) The theta series of $\mathcal{L}(C)$ and $\mathcal{L}^{+}(C)$ are given as follows:

$$
\begin{gathered}
\Theta_{\mathcal{L}(C)}(q)=W_{C}\left(\theta_{3}(q), \theta_{2}(q)\right), \\
\Theta_{\mathcal{L}^{+}(C)}(q)=\frac{1}{2}\left(W_{C}\left(\theta_{3}(q), \theta_{2}(q)\right)+\theta_{4}(q)^{n}\right), \\
\text { where } \theta_{3}(q)=\sum_{i \in \mathbb{Z}} q^{i^{2}}, \theta_{2}(q)=\sum_{i \in \mathbb{Z}} q^{(i+1 / 2)^{2}} \text { and } \theta_{4}(q)=\sum_{i \in \mathbb{Z}}(-1)^{i} q^{i^{2}} .
\end{gathered}
$$

Let us consider some examples. For (3), see [KKM91, (5.3.1)].

\section{Lemma 1.9.}

(1) The even lattice $\mathcal{L}\left(e_{8}\right)$ is isomorphic to $E_{8}$.

(2) The lattices $\mathcal{L}\left(e_{8}^{2}\right)$ and $\mathcal{L}\left(d_{16}^{+}\right)$are isomorphic to $E_{8}^{2}$ and $D_{16}^{+}$, respectively.

(3) The lattices $\mathcal{L}^{+}\left(e_{8}^{2}\right)$ and $\mathcal{L}^{+}\left(d_{16}^{+}\right)$are isomorphic.

Finally, we consider the VOAs $V_{L}$ and $V_{L}^{+}$of central charge $n$ obtained from an even lattice $L$ of rank $n$ ([Bo86, FLM88]).

Set $\mathfrak{h}_{L}=\mathbb{C} \otimes_{\mathbb{Z}} L$ and extend $(\cdot, \cdot)$ to a symmetric $\mathbb{C}$-bilinear form on $\mathfrak{h}_{L}$. We view $\mathfrak{h}_{L}=\mathbb{C} \otimes_{\mathbb{Z}} L$ as an abelian Lie algebra. Let $\hat{\mathfrak{h}}_{L}=\mathfrak{h}_{L} \otimes \mathbb{C}\left[t, t^{-1}\right] \oplus \mathbb{C}$ be its affine Lie algebra. Set $\hat{\mathfrak{h}}_{L}^{-}=\mathfrak{h}_{L} \otimes t^{-1} \mathbb{C}\left[t^{-1}\right]$ and let $S\left(\hat{\mathfrak{h}}_{L}^{-}\right)$be the symmetric algebra of 
$\hat{\mathfrak{h}}_{L}^{-}$. Then $S\left(\hat{\mathfrak{h}}_{L}^{-}\right) \cong \mathbb{C}\left[\alpha \otimes t^{n} \mid \alpha \in \mathfrak{h}_{L}, n<0\right] \cdot \mathbf{1}$ is the unique irreducible $\hat{\mathfrak{h}}_{L}$-module such that $\mathbf{c}$ acts as 1 and $\alpha \otimes t^{n} \cdot \mathbf{1}=0$ if $\alpha \in \mathfrak{h}_{L}$ and $n \geq 0$.

Let us consider the twisted group algebra of the additive group $L$. Let $\left\langle\kappa_{L}\right\rangle$ be a cyclic group of order 2 and let

$$
1 \rightarrow\left\langle\kappa_{L}\right\rangle \rightarrow \hat{L} \stackrel{\rho}{\rightarrow} L \rightarrow 1
$$

be a central extension of $L$ by $\left\langle\kappa_{L}\right\rangle$ with the commutator map $c_{0}(\alpha, \beta)=(\alpha, \beta)$ $(\bmod 2), \alpha, \beta \in L$. Then we obtain the twisted group algebra $\mathbb{C}\{L\}=\mathbb{C}[\hat{L}] /$ $\left(\kappa_{L}+1\right)$, where $\mathbb{C}[\hat{L}]$ is the usual group algebra of the group $\hat{L}$. The lattice VOA $V_{L}$ associated with $L$ is defined to be $V_{L}=S\left(\hat{\mathfrak{h}}_{L}^{-}\right) \otimes \mathbb{C}\{L\}$ as a vector space. We refer to [FLM88 for the definition of the vertex operator on $V_{L}$. Note that the central charge of $V_{L}$ is $n$.

Let $\theta_{V_{L}}$ be an automorphism of $V_{L}$ of order 2 which is a lift of the -1-isometry of $L$. Let $V_{L}^{+}$denote the subspace of $V_{L}$ fixed by $\theta_{V_{L}}$. Then $V_{L}^{+}$is a vertex operator subalgebra of $V_{L}$. Note that the automorphism $\theta_{V_{L}}$ is not unique, but the isomorphism type of $V_{L}^{+}$is independent of the choice of $\theta_{V_{L}}$ [DGH98, Appendix D].

As a summary, we obtain two VOAs of central charge $n$ :

$$
V_{L}=S\left(\hat{\mathfrak{h}}_{L}^{-}\right) \otimes \mathbb{C}\{L\} \quad \text { and } \quad V_{L}^{+}=\left\{v \in V_{L} \mid \theta_{V_{L}}(v)=v\right\} .
$$

We call the constructions of $V_{L}$ and $V_{L}^{+}$from an even lattice $L$ Construction $A$ and Construction $B$ respectively.

Lemma 1.10 (Do93, FLM88]). Let $L$ be an even lattice of rank $n$.

(1) If $L$ is unimodular, then $V_{L}$ is holomorphic.

(2) The characters of $V_{L}$ and $V_{L}^{+}$are given as follows:

$$
\begin{aligned}
\operatorname{ch}_{V_{L}}(q) & =\frac{\Theta_{L}(q)}{\eta(q)^{n}}, \\
\operatorname{ch}_{V_{L}^{+}}(q) & =\frac{1}{2}\left(\frac{\Theta_{L}(q)}{\eta(q)^{n}}+\frac{\eta(q)^{n}}{\eta(2 q)^{n}}\right),
\end{aligned}
$$

where $\eta(q)=q^{1 / 24} \prod_{i=1}^{\infty}\left(1-q^{i}\right)$.

The following lemma was verified in Sh06, Lemma 3.4].

Lemma 1.11. The VOAs $V_{E_{8}^{2}}^{+}$and $V_{D_{16}^{+}}^{+}$are isomorphic.

Now, we recall some facts about irreducible modules for $V_{L}^{+}$(FLM88, DN99, AD04). Let $V_{L}^{-}$be the -1-eigenspace of $\theta_{V_{L}}$ in $V_{L}$. Then $V_{L}^{-}$is an irreducible $V_{L}^{+}$-module. For a coset $\lambda+L \in L^{*} / L, V_{\lambda+L}=S\left(\hat{\mathfrak{h}}_{L}^{-}\right) \otimes \mathbb{C}[\lambda+L]$ is an irreducible $V_{L}$-module. If $\lambda \notin L / 2$, then $V_{\lambda+L}$ is also irreducible for $V_{L}^{+}$. If $\lambda \in L / 2$, then $V_{\lambda+L}$ is the direct sum of two irreducible $V_{L}^{+}$-submodules $V_{\lambda+L}^{+}$and $V_{\lambda+L}^{-}$. Set $J=\left\{a^{-1} \theta_{V_{L}}(a) \mid a \in \hat{L}\right\}$. Then $J$ is a normal subgroup of $\hat{L}$. Let $T_{\chi}$ be an irreducible module for $\hat{L} / J$ with character $\chi$ satisfying $\chi(\kappa J)=-1$. Then there are two irreducible $V_{L}^{+}$-modules $V_{L}^{T_{\chi}, \pm}$ associated with $T_{\chi}$.

It was shown in DN99, AD04 that any irreducible $V_{L}^{+}$-module is isomorphic to one of $V_{\lambda+L}^{ \pm}\left(\lambda \in L^{*} \cap(L / 2)\right), V_{\mu+L}\left(\mu \in L^{*} \backslash(L / 2)\right)$ and $V_{L}^{T_{\chi}, \pm}$. By the fusion rules for irreducible $V_{L}^{+}$-modules Ab01, ADL05, one can obtain the following lemma.

Lemma 1.12. The irreducible $V_{L}^{+}$-module $V_{\mu+L}\left(\mu \in L^{*} \backslash(L / 2)\right)$ is not a simple current. 
The characters of $V_{\lambda+L}^{ \pm}$and $V_{L}^{T_{\chi}, \pm}$ are given as follows ([FLM88]):

$$
\begin{aligned}
\operatorname{ch}_{V_{\lambda+L}^{ \pm}}(q) & =\frac{1}{2}\left(\frac{\Theta_{\lambda+L}(q)}{\eta(q)^{n}}\right) \quad(\lambda \notin L), \\
\operatorname{ch}_{V_{L}^{T \chi}, \pm}(q) & =\frac{\operatorname{dim} T_{\chi}}{2}\left(\frac{\eta(q)^{n}}{\eta\left(q^{1 / 2}\right)^{n}} \pm \frac{\eta\left(q^{2}\right)^{n} \eta\left(q^{1 / 2}\right)^{n}}{\eta(q)^{2 n}}\right) .
\end{aligned}
$$

2. Isomorphism problems for the EVEn lattices $\mathcal{L}(C)$ And $\mathcal{L}^{+}(C)$

2.1. A characterization of doubly-even binary codes obtained from Kleinian codes. In this subsection, we characterize the doubly-even binary codes obtained by Construction B from Kleinian codes, which will play an important role in Sections 2.3 and 2.4. Note that this is a code analogue of the characterization of the even lattices obtained by Construction B (see Proposition 3.2) given in Sh06.

Let $u_{i}=\left(0^{4(i-1)} 1^{4} 0^{n-4 i}\right) \in \mathbb{F}_{2}^{n}$ for $1 \leq i \leq n / 4$. If $m=n / 4 \in \mathbb{Z}$, then $d_{4}^{m}=\operatorname{Span}_{\mathbb{F}_{2}}\left\{u_{i} \mid 1 \leq i \leq m\right\}$ and $\left(d_{4}^{m}\right)_{0}=\operatorname{Span}_{\mathbb{F}_{2}}\left\{u_{i}+u_{j} \mid 1 \leq i, j \leq m\right\}$. For our purpose, we need the following lemma.

Lemma 2.1. Let $C$ be a doubly-even binary code of length $n=4 m \in 4 \mathbb{Z}$.

(1) If $d_{4}^{m} \subset C$, then $C \cong \mathcal{C}(K)$ for some even Kleinian code $K$ of length $m$.

(2) Assume that $\left(d_{4}^{m}\right)_{0} \subset C, u_{1} \notin C$ and $u_{1} \in C^{\perp}$. Then $C \cong \mathcal{C}^{+}(K)$ and $C+\mathbb{F}_{2} u_{1} \cong \mathcal{C}(K)$ for some even Kleinian code $K$ of length $m$.

Proof. (1) Let $x=\left(x_{1}, x_{2}, \ldots, x_{m}\right) \in C$, where $x_{i} \in \mathbb{F}_{2}^{4}$. Since $C$ is doubly even, $\left\langle x, u_{i}\right\rangle=\left\langle x_{i},(1111)\right\rangle=0$; equivalently, wt $\left(x_{i}\right) \in 2 \mathbb{Z}$. It follows from $\hat{\mathbb{K}}+\mathbb{F}_{2}(1111)=$ $\left\{v \in \mathbb{F}_{2}^{4} \mid \operatorname{wt}(v) \in 2 \mathbb{Z}\right\}$ that $x_{i} \in \hat{\mathbb{K}}$ or $x_{i}+(1111) \in \hat{\mathbb{K}}$. For the map ^, see (1.1). Hence there is a unique $k_{x} \in \mathbb{K}^{m}$ such that $x \in \hat{k}_{x}+d_{4}^{m}$. Set $K=\left\{k_{x} \mid x \in C\right\}$. Then $C \subset \mathcal{C}(K)$. It follows from $d_{4}^{m} \subset C$ that $\hat{k}_{x} \in C$. Thus by (1.1) $C \cong \mathcal{C}(K)$. Since $C$ is a doubly-even binary code, $K$ is an even Kleinian code.

(2) Set $\tilde{C}=C+\mathbb{F}_{2} u_{1}$. It follows from $u_{1} \in C^{\perp}$ and $\operatorname{wt}\left(u_{1}\right)=4$ that $\tilde{C}$ is doubly-even. Since $u_{1} \notin C$, we have $C \subsetneq \tilde{C}$. Let $y=\left(y_{1}, y_{2}, \ldots, y_{m}\right) \in C^{\perp} \backslash \tilde{C}^{\perp}$, where $y_{i} \in \mathbb{F}_{2}^{4}$. It follows from $\left\langle y, u_{i}\right\rangle \neq 0$ and $\left\langle y, u_{i}+u_{j}\right\rangle=0$ for all $i, j$ that $\operatorname{wt}\left(y_{i}\right)=1$ or 3 for all $i$. Since $\tilde{C}$ is doubly-even, $\tilde{C} \subset \tilde{C}^{\perp}$. Hence $u_{i} \in \tilde{C}^{\perp}$ for any $i$, and we may assume that $\operatorname{wt}\left(y_{i}\right)=1$ for all $i$, and $y=(00010001 \ldots 0001)$ up to coordinate permutation.

Let $x=\left(x_{1}, x_{2}, \ldots, x_{m}\right) \in C$, where $x_{i} \in \mathbb{F}_{2}^{4}$. Since $u_{i} \in C^{\perp}$ for all $i$, we have $\left\langle x_{i},(1111)\right\rangle=0$. Hence there is $k_{x} \in \mathbb{K}^{m}$ such that $x \in \hat{k}_{x}+d_{4}^{m}$. Since $\langle x, y\rangle=\sum_{i=1}^{m}\left\langle x_{i},(0001)\right\rangle=0$ and $\left\langle\hat{k}_{x}, y\right\rangle=0$, we have $x \in \hat{k}_{x}+\left(d_{4}^{m}\right)_{0}$. By the same argument as in (1), $K=\left\{k_{x} \mid x \in C\right\}$ is an even Kleinian code and $C \cong \mathcal{C}^{+}(K)$ (cf. (1.2) $)$. Moreover, $C+\mathbb{F}_{2} u_{1} \cong \mathcal{C}(K)$.

Proposition 2.2. Let $C$ be a doubly-even binary code of length $n$. If there exists a coset $x+C \in C^{\perp} / C$ satisfying $|(x+C)(4)| \geq n / 4+|C(4)|$, then $C \cong \mathcal{C}^{+}(K)$ and $C+\mathbb{F}_{2} x \cong \mathcal{C}(K)$ for some even Kleinian code $K$ of length $n / 4$.

Proof. In order to apply Lemma 2.1 (2), we will show that the coset $x+C$ contains $\left\{u_{i} \mid 1 \leq i \leq n / 4\right\}$. Assume $\left\{u_{i} \mid 1 \leq i \leq r\right\} \subset x+C$ and $r<n / 4$. We claim that the $\operatorname{coset} x+C$ contains $\left(0^{4 r}, z\right)$ with wt $(z)=4$.

Let $v=\left(v_{1}, v_{2}, \ldots, v_{r}, z\right) \in(x+C)(4)$, where $v_{i} \in \mathbb{F}_{2}^{4}$ and $z \in \mathbb{F}_{2}^{n-4 r}$. Then $v+u_{i} \in C$. It follows from $u_{i} \in C^{\perp}$ that $\left\langle v+u_{i}, u_{i}\right\rangle=\left\langle v_{i},(1111)\right\rangle=0$; equivalently, 
$\operatorname{wt}\left(v_{i}\right) \in 2 \mathbb{Z}$. If $\operatorname{wt}\left(v_{i}\right)=4$ for some $i$, then $v=u_{i}$ since $\operatorname{wt}(v)=4$. Set

$$
Y=\left\{\left(y_{1}, y_{2}, \ldots, y_{r}, z\right) \in(x+C)(4) \mid \operatorname{wt}\left(y_{i}\right)=2 \text { for some } 1 \leq i \leq r\right\} .
$$

For $y \in Y$, let $m(y)=\min \left\{i \mid \operatorname{wt}\left(y_{i}\right)=2\right\}$. In order to give an upper bound for $|Y|$, we consider the map $\psi: Y \rightarrow C(4), y \mapsto y+u_{m(y)}$ and show its injectivity. Assume $\psi(y)=\psi\left(y^{\prime}\right)$. Then $y+u_{m(y)}=y^{\prime}+u_{m\left(y^{\prime}\right)}$; equivalently, $y+y^{\prime}=u_{m(y)}+u_{m\left(y^{\prime}\right)}$. By the definition of $u_{i}, \operatorname{wt}\left(u_{m(y)}+u_{m\left(y^{\prime}\right)}\right)=0$ or 8 . If $\operatorname{wt}\left(u_{m(y)}+u_{m\left(y^{\prime}\right)}\right)=8$, then $m(y) \neq m\left(y^{\prime}\right)$. However, by $\operatorname{wt}\left(y+y^{\prime}\right)=8$, we have $\operatorname{wt}\left(y_{m(y)}\right)=\operatorname{wt}\left(y_{m\left(y^{\prime}\right)}\right)=$ $\operatorname{wt}\left(y_{m(y)}^{\prime}\right)=\operatorname{wt}\left(y_{m\left(y^{\prime}\right)}^{\prime}\right)=2$, and $m(y)=m\left(y^{\prime}\right)$, which is a contradiction. Hence $\operatorname{wt}\left(u_{m(y)}+u_{m\left(y^{\prime}\right)}\right)=0$; equivalently, $y=y^{\prime}$. Thus $\psi$ is injective. In particular, $|Y| \leq|C(4)|$.

By the definition of $Y$, we have

$$
\left\{(0, \ldots, 0, z) \in(x+C)(4) \mid z \in \mathbb{F}_{2}^{n-4 r}\right\}=(x+C)(4) \backslash\left(Y \cup\left\{u_{i} \mid 1 \leq i \leq r\right\}\right) .
$$

By the upper bound of $|Y|$,

$$
\left|(x+C)(4) \backslash\left(Y \cup\left\{u_{i} \mid 1 \leq i \leq r\right\}\right)\right| \geq|(x+C)(4)|-|C(4)|-r,
$$

and it is greater than 0 by $r<n / 4$ and the claim follows. Hence there is an element $\left(0^{4 r}, z\right) \in x+C$ with $\operatorname{wt}(z)=4$. We define it to be $u_{r+1}$ up to coordinate permutation. Thus the coset $x+C$ contains $\left\{u_{i} \mid 1 \leq i \leq n / 4\right\}$ up to coordinate permutation, and $n / 4$ must be an integer. Moreover, $u_{i}+u_{j} \in C$ for all $i, j$. Hence $u_{1} \in C^{\perp}, u_{1} \notin C$, and $\operatorname{Span}_{\mathbb{F}_{2}}\left\{u_{i}+u_{j} \mid 1 \leq i, j \leq m\right\}=\left(d_{4}^{m}\right)_{0} \subset C$. Therefore the proposition follows from Lemma $2.1(2)$.

Remark 2.3. If $C=\mathcal{C}^{+}(K)$, then $\left|\left(\left(1^{4} 0^{n-4}\right)+C\right)(4)\right|=n / 4+|C(4)|$.

2.2. Even lattices $\mathcal{L}(C)$. In this subsection, we study the isomorphism classes of $\mathcal{L}(C)$. The following proposition is well known (cf. CS99, Chapter 18, Proposition 4]). However, we give a sketch of a proof since it would be useful for understanding the arguments in the VOA case (see Section 3.1).

Proposition 2.4. Let $C$ and $D$ be doubly-even binary codes of length $n$. Then $\mathcal{L}(C) \cong \mathcal{L}(D)$ if and only if $C \cong D$.

Proof. The "if" part is obvious. For the "only" part, we assume that $\mathcal{L}(C) \cong$ $\mathcal{L}(D)$. One can verify that the Weyl group of the norm 2 vectors in $\mathcal{L}(C)$ is transitive on the set of all orthogonal bases of norm 2 in $\mathcal{L}(C)$. Hence we may assume that the canonical bases $\left\{\alpha_{i} \mid 1 \leq i \leq n\right\}$ of $\mathcal{L}(C)$ and $\mathcal{L}(D)$ in (1.3) are the same up to automorphism. Since the binary code $C$ can be recovered from $\left\{\alpha_{i} \mid 1 \leq i \leq n\right\}$, we obtain $C \cong D$.

2.3. Even lattices $\mathcal{L}^{+}(C)$. In this subsection, we study the isomorphism classes of $\mathcal{L}^{+}(C)$.

The following lemma can be easily proved by using the quadratic spaces of plus type over $\mathbb{F}_{2}$ associated to doubly-even binary codes of length $8 k$ containing $\left(1^{8 k}\right)$.

Lemma 2.5. For any doubly-even binary code of length $8 k$, there exists a doublyeven self-dual binary code of length $8 k$ containing it.

By (1.2),$\left(d_{4}^{m}\right)_{0} \subset \mathcal{C}^{+}(K)$ and $(00010001 \ldots 0001) \in \mathcal{C}^{+}(K)^{\perp}$. Hence by [KKM91, Lemma 3.3.2], we obtain the following lemma. 
Lemma 2.6. Let $K$ be an even Kleinian code of length $m$. Let $\rho$ be the orthogonal transformation of $\mathbb{R}^{4 m}$ defined by

$$
\rho\left(\alpha_{j}\right)=\left\{\begin{array}{cl}
\frac{1}{2}\left(\alpha_{4 i-3}+\alpha_{4 i-2}+\alpha_{4 i-1}+\alpha_{4 i}\right) & \text { if } j=4 i, \\
\frac{1}{2}\left(-\alpha_{4 i-3}-\alpha_{4 i-2}-\alpha_{4 i-1}+\alpha_{4 i}\right)+\alpha_{j} & \text { if } 4 i-3 \leq j \leq 4 i-1,
\end{array}\right.
$$

where $\left\{\alpha_{i} \mid 1 \leq i \leq 4 m\right\}$ is the basis of $\mathbb{R}^{4 m}$ as defined in (1.3) and (1.4). Then $\rho$ is an isomorphism of lattices from $\mathcal{L}\left(\mathcal{C}^{+}(K)\right)$ to $\mathcal{L}^{+}(\mathcal{C}(K))$.

The following proposition was shown in KKM91 if $n>16$, and the case $n \leq 16$ is a finite problem. However, we give a proof here since it is useful for understanding the VOA case (Theorem 3.9).

Theorem 2.7 (cf. [KKM91, Theorem 3]). Let $C$ and $D$ be doubly-even binary codes of length $n$. Then $\mathcal{L}^{+}(C) \cong \mathcal{L}^{+}(D)$ if and only if one of the following holds.

(1) $C$ and $D$ are equivalent.

(2) $C$ and $D$ are doubly-even self-dual binary codes of length 16.

Proof. The "if" part follows from Lemmas 1.2 (2) and 1.9 (3). For the "only" part, we assume that $\mathcal{L}^{+}(C) \cong \mathcal{L}^{+}(D)$. Then by Lemma 1.8 (3) and the fact that $\theta_{2}$ and $\theta_{3}$ are algebraically independent (Eb02, Section 2.9]), $W_{C}(X, Y)=$ $W_{D}(X, Y)$. Since $\mathcal{L}^{+}(C) \cong \mathcal{L}^{+}(D)$, there is an isomorphism $\varphi: \mathcal{L}^{+}(D)^{*} / \mathcal{L}^{+}(D) \rightarrow$ $\mathcal{L}^{+}(C)^{*} / \mathcal{L}^{+}(C)$ of their discriminant groups. Let $\lambda+\mathcal{L}^{+}(C)=\varphi\left(\alpha_{1}+\mathcal{L}^{+}(D)\right)$, where $\left\{\alpha_{i} \mid 1 \leq i \leq n\right\}$ is the canonical basis of $\mathbb{R}^{n}$ as defined in (1.3) and (1.4). Without loss of generality, we assume that $\langle\lambda, \lambda\rangle=2$. By (1.4),

$$
\mathcal{L}^{+}(C)^{*}=\mathcal{L}\left(C^{\perp}\right)+\mathbb{Z} \frac{1}{4} \alpha_{\left(1^{n}\right)} .
$$

For $c=\left(c_{i}\right) \in \mathbb{F}_{2}^{n}$, let $\varepsilon_{c}$ be the orthogonal transformation defined by $\varepsilon_{c}\left(\alpha_{i}\right)=$ $(-1)^{c_{i}} \alpha_{i}$. Then $\varepsilon_{c}$ is an automorphism of $\mathcal{L}^{+}(C)$ if $c \in C^{\perp}$. By the actions of $\varepsilon_{c}$, we may assume that $\lambda=\alpha_{1}, \alpha_{y} / 2\left(y \in C^{\perp}(4) \backslash C\right), \alpha_{\left(1^{n}\right)} / 4-\alpha_{1}$ or $\alpha_{\left(1^{n}\right)} / 4$.

(i) Assume that $\lambda=\alpha_{1}$. Then $\mathcal{L}(C) \cong \mathcal{L}(D)$, and by Proposition 2.4. $C \cong D$.

(ii) Assume that $\lambda=\alpha_{y} / 2$. Comparing the numbers of vectors of norm 2 in $\alpha_{1}+$ $\mathcal{L}^{+}(D)$ and $\lambda+\mathcal{L}^{+}(C)$, we obtain $2^{3}|D(4)|+2 n=2^{3}|(y+C)(4)|$. By $W_{C}(X, Y)=$ $W_{D}(X, Y)$, we have $|C(4)|=|D(4)|$. Combining the equations, we obtain

$$
2^{3}|C(4)|+2 n=2^{3}|(y+C)(4)| .
$$

Then by Proposition 2.2 $C \cong \mathcal{C}^{+}(K)$ and $C+\mathbb{F}_{2} y \cong \mathcal{C}(K)$ for some even Kleinian code $K$ of length $n / 4$. Hence

$$
\mathcal{L}(D)=\mathcal{L}^{+}(D) \cup\left(\alpha_{1}+\mathcal{L}^{+}(D)\right) \cong \mathcal{L}^{+}(C) \cup\left(\lambda+\mathcal{L}^{+}(C)\right) \cong \mathcal{L}^{+}(\mathcal{C}(K)) \cong \mathcal{L}(C),
$$

where the last isomorphism is given in Lemma 2.6. Thus by Proposition 2.4, $C \cong D$.

(iii) Assume that $\lambda=\alpha_{\left(1^{n}\right)} / 4-\alpha_{1}$. Since the norm of $\lambda$ is $2, n$ must be 8 . Since $2 \lambda \in \mathcal{L}^{+}(C), C$ contains $\left(1^{8}\right)$. It follows from $W_{C}(X, Y)=W_{D}(X, Y)$ that $D$ also has $\left(1^{8}\right)$ and $\operatorname{dim} C=\operatorname{dim} D$. One easily sees that there are only four doubly-even binary codes of length 8 containing $\left(1^{8}\right)$ up to equivalence, and those are determined by the dimensions. Hence $C \cong D$.

(iv) Assume that $\lambda=\alpha_{\left(1^{n}\right)} / 4$. Since the norm of $\lambda$ is $2, n$ must be 16. Let $k$ be the dimension of $C$. Then $C^{\perp} / C \cong \mathbb{Z}_{2}^{16-2 k}$. By $\left|\left(\alpha_{1}+\mathcal{L}^{+}(C)\right)(2)\right|=\mid(\lambda+$ $\left.\mathcal{L}^{+}(C)\right)(2) \mid$, we obtain $2^{3}|C(4)|+2^{5}=2^{k}$; equivalently,

$$
|C(4)|=2^{k-3}-4 .
$$


If $C$ is self-dual, then so is $D$, and hence (2) holds. Assume that $C$ is not self-dual. Then by $|C(4)| \geq 0$, we have $5 \leq k \leq 7$. By Lemma 2.5, there is a doubly-even self-dual binary code $E$ of length 16 such that $C \subsetneq E$. By Lemma $1.3(2), E \cong d_{16}^{+}$ or $e_{8}^{2}$, and $|E(4)|=28$. Hence there exists a coset $x+C \in E / C$ such that

$$
|(x+C)(4)| \geq \frac{|E(4)|-|C(4)|}{|E / C|-1}=\frac{2^{5}-2^{k-3}}{2^{8-k}-1}=2^{k-3}=|C(4)|+\frac{16}{4} .
$$

By Proposition 2.2, $C \cong \mathcal{C}^{+}(K)$ for some even Kleinian code $K$ of length 4 . By $\left|\mathcal{C}^{+}(K)\right|=2^{3}|K|$, we have $|K|=2^{k-3}$. By Lemma 1.6 (3) and (2.1), $|K(2)|=$ $|C(4)| / 2=2^{k-4}-2$. Since $|K(4)|=|K|-|K(2)|-|K(0)|=2^{k-4}+1$, we obtain

$$
W_{K}(X, Y)=\left(2^{k-4}+1\right) X^{4}+\left(2^{k-4}-2\right) X^{2} Y^{2}+Y^{4} .
$$

Replacing $C$ by $D$, we also have $C \cong D$, or $D \cong \mathcal{C}^{+}(J)$ for some even Kleinian code $J$ of length 4 with the same properties as $K$. One can verify that there are only four even Kleinian codes of length 4 satisfying (2.2) up to equivalence (cf. Hö03a ). Two of them are characterized by the weight enumerators, and the others are the two even self-dual Kleinian codes $\epsilon_{2}^{2}$ and $\delta_{4}^{+}$of length 4 (Lemma 1.1 (2)). By Lemma $1.7(3), \mathcal{C}^{+}\left(\epsilon_{2}^{2}\right) \cong \mathcal{C}^{+}\left(\delta_{4}^{+}\right)$. Thus we obtain $C \cong D$.

2.4. Even lattices $\mathcal{L}(C)$ and $\mathcal{L}^{+}(C)$. In this subsection, we study the isomorphism classes of $\mathcal{L}(C)$ and $\mathcal{L}^{+}(C)$.

Theorem 2.8. Let $C$ and $D$ be doubly-even binary codes of length $n$. Then $\mathcal{L}^{+}(C) \cong \mathcal{L}(D)$ if and only if there exists an even Kleinian code $K$ such that $C \cong \mathcal{C}(K)$ and $D \cong \mathcal{C}^{+}(K)$.

Proof. The "if" part follows from Lemma 2.6. For the "only" part, we assume that $\mathcal{L}^{+}(C) \cong \mathcal{L}(D)$. Comparing the numbers of vectors of norm 2 in the lattices (Lemma $1.8(3)$ ), we obtain

$$
8|C(4)|=2 n+16|D(4)| .
$$

Since $\mathcal{L}^{+}(C) \cong \mathcal{L}(D)$, there is an isomorphism $\varphi: \mathcal{L}^{+}(C)^{*} / \mathcal{L}^{+}(C) \rightarrow \mathcal{L}(D)^{*} / \mathcal{L}(D)$ of their discriminant groups. By $\mathcal{L}(D)^{*}=\mathcal{L}\left(D^{\perp}\right)$, there is an $x \in D^{\perp} \backslash D$ such that $\alpha_{x} / 2+\mathcal{L}(D)=\varphi\left(\alpha_{1}+\mathcal{L}^{+}(C)\right)$. Comparing the numbers of vectors of norm 2 in $\alpha_{1}+\mathcal{L}^{+}(C)$ and $\alpha_{x} / 2+\mathcal{L}(D)$, we obtain

$$
2 n+8|C(4)|=16|(x+D)(4)| .
$$

Combining the equations, we obtain

$$
|(x+D)(4)|=n / 4+|D(4)| .
$$

By Proposition $2.2, D \cong \mathcal{C}^{+}(K)$ for some even Kleinian code $K$. Then by Proposition 2.6 and an assumption,

$$
\mathcal{L}^{+}(\mathcal{C}(K)) \cong \mathcal{L}\left(\mathcal{C}^{+}(K)\right) \cong \mathcal{L}(D) \cong \mathcal{L}^{+}(C)
$$

By Theorem 2.7, $C \cong \mathcal{C}(K)$ or both $C$ and $\mathcal{C}(K)$ are (nonisomorphic) doubly-even self-dual binary codes of length 16 . In the first case, we are done. In the latter case, Lemmas 1.2 (2) and 1.7 (2) show that $C \cong \mathcal{C}\left(K^{\prime}\right)$, where $\left\{K, K^{\prime}\right\}=\left\{\epsilon_{2}^{2}, \delta_{4}^{+}\right\}$. By Lemma $1.7(3), \mathcal{C}^{+}(K) \cong \mathcal{C}^{+}\left(K^{\prime}\right)$. Hence we have $D \cong \mathcal{C}^{+}\left(K^{\prime}\right)$ and $C \cong \mathcal{C}\left(K^{\prime}\right)$. 


\section{IsOMORPHISM PROBLEMS FOR THE VOAs $V_{L}$ AND $V_{L}^{+}$}

3.1. VOAs $V_{L}$. In this subsection, we study the isomorphism classes of $V_{L}$. The following proposition is well known. Its proof is similar to that of Proposition 2.4.

Proposition 3.1. Let $L$ and $N$ be even lattices of rank $n$. Then $V_{L} \cong V_{N}$ if and only if $L \cong N$.

Proof. The "if" part is obvious. For the "only" part, we assume $V_{L} \cong V_{N}$. By the VOA structure of $V_{L}$ ([FLM88] $), S\left(\hat{\mathfrak{h}}_{L}^{-}\right)$is a subVOA and $V_{L} \cong \bigoplus_{\alpha \in L} S\left(\hat{\mathfrak{h}}_{L}^{-}\right) \otimes$ $e^{\alpha}$ as $S\left(\hat{\mathfrak{h}}_{L}^{-}\right)$-modules. Note that $\mathfrak{h}_{L}(-1) \mathbf{1}=S\left(\hat{\mathfrak{h}}_{L}^{-}\right) \cap\left(V_{L}\right)_{1}$. Then for $h \in \mathfrak{h}_{L}$, $S\left(\hat{\mathfrak{h}}_{L}^{-}\right) \otimes e^{\alpha}$ is the eigenspace of the 0 -th product of $h(-1) \mathbf{1}$ with eigenvalue $(\alpha, h)$. By the same argument, $V_{N} \cong \bigoplus_{\beta \in N} S\left(\hat{\mathfrak{h}}_{N}^{-}\right) \otimes e^{\beta}$ as $S\left(\hat{\mathfrak{h}}_{N}^{-}\right)$-modules, and $\mathfrak{h}_{N}(-1) \mathbf{1}=$ $S\left(\hat{\mathfrak{h}}_{N}^{-}\right) \cap\left(V_{N}\right)_{1}$. Since $\operatorname{dim}\left(V_{L}\right)_{0}=1,\left(V_{L}\right)_{1}$ has a finite-dimensional reductive Lie algebra structure by the 0 -th product. Using the fact that $V_{L} \cong V_{N}$, we may view both $\mathfrak{h}_{L}(-1) \mathbf{1}$ and $\mathfrak{h}_{N}(-1) \mathbf{1}$ as Cartan subalgebras of $\left(V_{L}\right)_{1}$. Hence there exists $g \in\left\langle\exp \left(v_{0}\right) \mid v \in\left(V_{L}\right)_{1}\right\rangle \subset \operatorname{Aut}\left(V_{L}\right)$ such that $g\left(\mathfrak{h}_{N}(-1) \mathbf{1}\right)=\mathfrak{h}_{L}(-1) \mathbf{1}$. Since $S\left(\hat{\mathfrak{h}}_{N}^{-}\right)$is generated by $\mathfrak{h}_{N}(-1) \mathbf{1}$ as a VOA, we have $g\left(S\left(\hat{\mathfrak{h}}_{N}^{-}\right)\right)=S\left(\hat{\mathfrak{h}}_{L}^{-}\right)$. Hence for any $\beta \in N, g\left(S\left(\hat{\mathfrak{h}}_{N}^{-}\right) \otimes e^{\beta}\right)=S\left(\hat{\mathfrak{h}}_{L}^{-}\right) \otimes e^{\alpha}$ for some $\alpha \in L$. Set $\bar{g}(\beta)=\alpha$. Since $g$ is an automorphism of a VOA, $\bar{g}$ is a linear isomorphism from $N$ to $L$, and $(\bar{g}(\beta), \bar{g}(\beta))=(\beta, \beta)$ for all $\beta \in N$. Thus $\bar{g}$ is an orthogonal transformation, and $N \cong L$.

3.2. VOAs $V_{L}^{+}$. In this subsection, we study the isomorphism classes of $V_{L}^{+}$.

The following proposition, which is similar to Proposition 2.2, was proved in Sh06.

Proposition 3.2 (Sh06, Theorem 2.2]). Let $L$ be an even lattice of rank $n$. If there exists $\lambda \in\left(L^{*} \cap L / 2\right)$ such that $|(\lambda+L)(2)| \geq 2 n+|L(2)|$, then $L \cong \mathcal{L}^{+}(C)$ and $L+\mathbb{Z} \lambda \cong \mathcal{L}(C)$ for some doubly-even binary code $C$.

Next, we will consider an even lattice $L$ such that $\sqrt{2} L^{*}$ is even.

Lemma 3.3. Let $L$ be an even lattice. If $\sqrt{2} L^{*}$ is even, then $2 L^{*} \subset L$.

Proof. Since $\sqrt{2} L^{*}$ is even, we have $\sqrt{2} L^{*} \subset\left(\sqrt{2} L^{*}\right)^{*}=L / \sqrt{2}$, and $2 L^{*} \subset L$.

Remark 3.4. In [Sh04, Sh06], an even lattice $L$ was said to be 2-elementary totally even if $2 L^{*} \subset L$ and if $\sqrt{2} L^{*}$ is even. By the lemma above, if $\sqrt{2} L^{*}$ is even, then $L$ is 2-elementary totally even.

The following lemma can be proved by using the quadratic spaces over $\mathbb{F}_{2}$ associated to 2-elementary totally even lattices of rank $8 k$. Note that it is of plus type by [Ve79, Theorem 1].

Lemma 3.5. Let $L$ be an even lattice of rank $8 k$ such that $\sqrt{2} L^{*}$ is even. Then there exists an even unimodular lattice of rank $8 k$ containing $L$.

Let us review some properties of irreducible modules for $V_{L}^{+}$.

Lemma 3.6. The following three conditions are equivalent.

(1) Any irreducible $V_{L}^{+}$-module is a self-dual simple current.

(2) An irreducible $V_{L}^{+}$-module $V_{L}^{T_{\chi}, \varepsilon}$ is self-dual.

(3) $\sqrt{2} L^{*}$ is even. 
Proof. Obviously, (1) shows (2). By Lemma 3.3 and ADL05, one can see that (3) implies (1).

Assume (2) and let $V_{L}^{T_{\chi}, \varepsilon}$ be a self-dual irreducible $V_{L}^{+}$-module. Let $M$ be the contragredient module of $V_{L}^{T_{\chi}, \varepsilon}$. Then by ADL05, Proposition 3.7], $M \cong V_{L}^{T_{\chi^{\prime}}, \varepsilon}$ and $\chi^{\prime}(s)=(-1)^{(\rho(s), \rho(s)) / 2} \chi(s)$ for $s \in Z(\hat{L} / J)$, where $\rho$ is given in (1.5). Since $V_{L}^{T_{\chi}, \varepsilon}$ is self-dual, we have $\chi^{\prime}=\chi$. It follows from $\rho(Z(\hat{L} / J))=2 L^{*} / 2 L$ that $(v, v) \in 4 \mathbb{Z}$ for all $v \in 2 L^{*}$. Hence $\sqrt{2} L^{*}$ is even, and we obtain (3).

Note that the fusion rules of $V_{L}^{+}$are associative [ADL05, Theorem 5.18].

Lemma 3.7 (cf. ADL05]). Let $L$ be an even lattice such that $\sqrt{2} L^{*}$ is even. Then the fusion group of $V_{L}^{+}$is isomorphic to an elementary abelian 2-group of order $2^{k+2}$, where $k$ is the integer satisfying $L^{*} / L \cong \mathbb{Z}_{2}^{k}$.

A triality automorphism of $V_{\mathcal{L}^{+}(C)}^{+}$which sends $V_{\mathcal{L}^{+}(C)}^{-}$to $V_{\alpha_{1}+\mathcal{L}^{+}(C)}^{+}$was given in [FLM88, Chapter 11]. The following lemma can be verified directly.

Lemma 3.8 (cf. [FLM88, Chapter 11]). Let $C$ be a doubly-even binary code. Then there is an isomorphism of VOAs from $V_{\mathcal{L}^{+}(C)}$ to $V_{\mathcal{L}(C)}^{+}$.

Now, we prove the following. Its proof is similar to that of Theorem 2.7

Theorem 3.9. Let $L$ and $N$ be even lattices of rank $n$. Then the VOAs $V_{L}^{+}$and $V_{N}^{+}$are isomorphic if and only if one of the following holds.

(1) $L$ and $N$ are isomorphic.

(2) $L$ and $N$ are even unimodular lattices of rank 16.

Proof. The "if" part follows from Lemmas 1.3 (2) and 1.11. For the "only" part, we assume that $V_{L}^{+} \cong V_{N}^{+}$. By Lemma 1.10 (2), $\Theta_{L}(q)=\Theta_{N}(q)$, and hence $\operatorname{ch} V_{L}^{-}=\operatorname{ch} V_{N}^{-}$. We regard the irreducible $V_{N}^{+}$-module $V_{N}^{-}$as an irreducible $V_{L}^{+}$module. Since $V_{N}^{-}$is a self-dual simple current, it is isomorphic to $V_{L}^{-}, V_{\lambda+L}^{\varepsilon}(\lambda \notin L)$ or $V_{L}^{T_{\chi}, \varepsilon}$ as $V_{L}^{+}$-modules under the isomorphism of $V_{N}^{+}$and $V_{L}^{+}$by Lemma 1.12

(i) Assume that $V_{N}^{-} \cong V_{L}^{-}$. Then $V_{N} \cong V_{L}^{+} \oplus V_{L}^{-}$is a simple current extension of $V_{L}^{+}$. Hence by Proposition 1.5, $V_{N} \cong V_{L}$ as VOAs, and by Proposition 3.1, $L \cong N$.

(ii) Assume that $V_{N}^{-} \cong V_{\lambda+L}^{\varepsilon}$. Since $\operatorname{Hom}\left(L^{*} / 2 L^{*}, \mathbb{Z}_{2}\right) \subset \operatorname{Aut}\left(V_{L}^{+}\right)($[FLM88] $)$, we may assume that $\varepsilon=+$ up to conjugation ([Sh04]). It follows from $\operatorname{ch} V_{L}^{-}=$ $\operatorname{ch} V_{N}^{-}$that $\operatorname{ch} V_{L}^{-}=\operatorname{ch} V_{\lambda+L}^{+}$. Comparing the dimensions of the weight 1 subspaces of $V_{L}^{-}$and $V_{\lambda+L}^{+}$(Lemma 1.10 and (1.6)), we obtain $|L(2)|+2 n=|(\lambda+L)(2)|$. Hence by Proposition 3.2, $L \cong \mathcal{L}^{+}(C)$ and $L+\mathbb{Z} \lambda \cong \mathcal{L}(C)$ for some doubly-even binary code $C$. By Proposition 1.5, as VOAs,

$$
V_{N} \cong V_{L}^{+} \oplus V_{N}^{-} \cong V_{L}^{+} \oplus V_{\lambda+L}^{+} \cong V_{\mathcal{L}(C)}^{+} \cong V_{L},
$$

where the last isomorphism is given in Lemma 3.8, By Proposition 3.1, we obtain $L \cong N$.

(iii) Assume that $V_{N}^{-} \cong V_{L}^{T_{\chi},-}$. By Lemma 1.10 and (1.7), the lowest weights of $V_{N}^{-}$and $V_{L}^{T_{\chi},-}$ are 1 and $n / 16+1 / 2$, respectively. Hence $n=8$. Since $V_{N}^{-}$is a selfdual, so is $V_{L}^{T_{\chi},-}$. Hence $\sqrt{2} L^{*}$ is even by Lemma3.6. Since $\sqrt{2}\left(\sqrt{2} L^{*}\right)^{*}=L$ is even, we have $\sqrt{2} L^{*} \subset E_{8}$ by Lemmas 1.3 (1) and 3.5. Hence $E_{8} \subset\left(\sqrt{2} L^{*}\right)^{*}=L / \sqrt{2}$; equivalently, $\sqrt{2} E_{8} \subset L$. Replacing $N$ by $L$, we have (i), (ii) or (iii) for $V_{L}^{-}$. The first two cases show that $L \cong N$. Hence we may assume that $\sqrt{2} E_{8} \subset N$ by the 
same argument. One easily checks that there are only five even overlattices of $\sqrt{2} E_{8}$ up to isomorphism, and those are determined by the discriminant groups. Since the fusion groups of $V_{L}^{+}$and $V_{N}^{+}$are isomorphic, so are the discriminant groups of $L$ and $N$ by Lemma 3.7. Thus $L \cong N$.

(iv) Assume that $V_{N}^{-} \cong V_{L}^{T_{\chi},+}$. By (1.7), the lowest weight of $V_{L}^{T_{\chi},+}$ is $n / 16$. By the same argument as in (iii), $\sqrt{2} L^{*}$ is even and $n=16$. Comparing the dimensions of the weight 1 subspaces of $V_{N}^{-}$and $V_{L}^{T_{\chi},+}$ (Lemma 1.10 and (1.7)), we obtain

$$
|L(2)|=2^{9-k}-32,
$$

where $\left|L^{*} / L\right|=2^{2 k}$. If $L$ is unimodular, then so is $N$, and hence (2) holds. Assume that $L$ is not unimodular. By Lemma 3.5 there exists an even unimodular lattice $P$ of rank 16 such that $L \subsetneq P$. By Lemma $1.3(2),|P(2)|=480$. Hence there exists a coset $\mu+L \in P / L$ such that

$$
|(\mu+L)(2)| \geq \frac{|P(2)|-|L(2)|}{|P / L|-1}=\frac{512-2^{9-k}}{2^{k}-1}=2^{9-k}=|L(2)|+2 \times 16 .
$$

By Proposition 3.2, $L \cong \mathcal{L}^{+}(C)$ for some $t$-dimensional doubly-even binary code $C$ of length 16. It follows from $\left|\mathcal{L}^{+}(C)^{*} / \mathcal{L}^{+}(C)\right|=2^{18-2 t}$ that $t=9-k$. By Lemma 1.8 and $n=16,\left|\mathcal{L}^{+}(C)(2)\right|=2^{3}|C(4)|+2 \times 16$. Hence, by (3.1), we obtain

$$
|C(4)|=2^{t-3}-4 \text {. }
$$

Since $|C(4)| \geq 0$, we have $5 \leq t \leq 8$. By the same arguments as in (iv) of Theorem 2.7 (see (2.1)), if $C$ is not self-dual, then $C \cong \mathcal{C}^{+}(K)$ for some even Kleinian code $K$ of length 4 with the weight enumerator (2.2).

Replacing $L$ by $N$, we also obtain $L \cong N$ or $N \cong \mathcal{L}^{+}(D)$ for some doubly-even binary code $D$ of length 16 with the same properties as $C$. If $C$ is self-dual, then so is $D$, and $L \cong N$ by Lemma 1.9 (3). If not, $C \cong \mathcal{C}^{+}(K)$ and $D \cong \mathcal{C}^{+}(J)$ for some even Kleinian codes $K$ and $J$ of length 4 . By Lemma 3.7 we have $L^{*} / L \cong N^{*} / N$, $\operatorname{dim} C=\operatorname{dim} D$, and $\operatorname{dim} K=\operatorname{dim} J$. Then by (2.2), both Kleinian codes $K$ and $J$ have the same weight enumerator. Thus $K \cong J$ or $\{K, J\}=\left\{\varepsilon_{2}^{2}, \delta_{4}^{+}\right\}$(cf. [Hö03a]). Therefore by Lemma 1.7, $C \cong D$, and $L \cong \mathcal{L}^{+}(C) \cong \mathcal{L}^{+}(D) \cong N$.

3.3. VOAs $V_{L}$ and $V_{L}^{+}$. In this subsection, we study the isomorphism classes of $V_{L}$ and $V_{L}^{+}$. The proof of the following theorem is similar to that of Theorem 2.8,

Theorem 3.10. Let $L$ and $N$ be even lattices of rank $n$. Then the VOAs $V_{L}^{+}$and $V_{N}$ are isomorphic if and only if there exists a doubly-even binary code $C$ such that $L \cong \mathcal{L}(C)$ and $N \cong \mathcal{L}^{+}(C)$.

Proof. Lemma 3.8 proves the "if" part. For the "only" part, we assume that $V_{L}^{+} \cong V_{N}$. Comparing the dimensions of the weight 1 subspaces of $V_{L}^{+}$and $V_{N}$ (Lemma $1.10(2)$ ), we obtain

$$
\frac{|L(2)|}{2}=n+|N(2)| .
$$

Let $V_{\lambda+N}$ be an irreducible $V_{N}$-module such that $V_{\lambda+N} \cong V_{L}^{-}$under the isomorphism of $V_{N}$ and $V_{L}^{+}$. Comparing the dimensions of the weight 1 subspaces of $V_{L}^{-}$ and $V_{\lambda+N}$, we obtain

$$
\frac{|L(2)|}{2}+n=|(\lambda+N)(2)| .
$$


Combining the equations, we obtain

$$
|N(2)|+2 n=|(\lambda+N)(2)|
$$

In addition, the fusion rule $\left[V_{L}^{-}\right] \times\left[V_{L}^{-}\right]=\left[V_{L}^{+}\right]$(ADL05) shows that $\left[V_{\lambda+N}\right] \times$ $\left[V_{\lambda+N}\right]=\left[V_{N}\right]$. Hence $2 \lambda \in N$. By Proposition 3.2, $N \cong \mathcal{L}^{+}(C)$ for some doublyeven binary code $C$ of length $n$. By Lemma 3.8, we obtain $V_{L}^{+} \cong V_{N} \cong V_{\mathcal{L}^{+}(C)} \cong$ $V_{\mathcal{L}(C)}^{+}$as VOAs. By Theorem 3.9, $L \cong \mathcal{L}(C)$ or both $L$ and $\mathcal{L}(C)$ are (nonisomorphic) even unimodular lattices of rank 16 . In the first case, we are done. In the latter case, Lemmas 1.3 (2) and 1.9 (2) show that $L \cong \mathcal{L}(D)$, where $\{C, D\}=\left\{e_{8}^{2}, d_{16}^{+}\right\}$. By Theorem 2.7. $\mathcal{L}^{+}(C) \cong \mathcal{L}^{+}(D)$. Hence $N \cong \mathcal{L}^{+}(D)$ and $L \cong \mathcal{L}(D)$.

\section{ACKNOWLEDGEMENTS}

The author thanks the referee for helpful suggestions and useful comments. He also thanks Professor Ching Hung Lam for helpful comments. Part of the work was done when the author was visiting Imperial College London in 2010. He thanks the staff for their help.

\section{REFERENCES}

[Ab01] T. Abe, Fusion rules for the charge conjugation orbifold, J. Algebra 242 (2001), 624655. MR 1848962 (2002f:17044)

[AD04] T. Abe and C. Dong, Classification of irreducible modules for the vertex operator algebra $V_{L}^{+}$: general case. J. Algebra 273 (2004), 657-685. MR2037717(2005c:17040)

[ADL05] T. Abe, C. Dong, and H. Li, Fusion rules for the vertex operator algebra $M(1)$ and $V_{L}^{+}$, Comm. Math. Phys. 253 (2005), 171-219. MR2105641 (2005i:17033)

[Bo86] R.E. Borcherds, Vertex algebras, Kac-Moody algebras, and the Monster, Proc. Nat'l. Acad. Sci. U.S.A. 83 (1986), 3068-3071. MR843307 (87m:17033)

[CS99] J.H. Conway and N.J.A. Sloane, Sphere packings, lattices and groups, 3rd Edition, Springer, New York, 1999. MR.1662447 (2000b:11077)

[Do93] C. Dong, Vertex algebras associated with even lattices, J. Algebra 161 (1993), 245-265. MR.1245855(94j:17023)

[DGH98] C. Dong, R.L. Griess, and G. Höhn, Framed vertex operator algebras, codes and Moonshine module, Comm. Math. Phys. 193 (1998), 407-448. MR.1618135 (99g:17050)

[DM04] C. Dong and G. Mason, Rational vertex operator algebras and the effective central charge, Int. Math. Res. Not. (2004), no. 56, 2989-3008. MR.2097833 (2005k:17034)

[DM04b] C. Dong and G. Mason, Holomorphic vertex operator algebras of small central charge, Pacific J. Math. 213 (2004), 253-266. MR2036919 (2005c:17044)

[DN99] C. Dong and K. Nagatomo, Representations of vertex operator algebra $V_{L}^{+}$for rank one lattice L, Comm. Math. Phys. 202 (1999), 169-195. MR1686535(2000b:17037)

[Eb02] W. Ebeling, Lattices and codes, Second revised edition, Advanced Lectures in Mathematics, Friedr. Vieweg \& Sohn, Braunschweig, 2002. MR1938666 (2003i:11093)

[FHL93] I. Frenkel, Y. Huang and J. Lepowsky, On axiomatic approaches to vertex operator algebras and modules, Mem. Amer. Math. Soc. 104 (1993). MR1142494(94a:17007)

[FLM88] I. Frenkel, J. Lepowsky and A. Meurman, Vertex operator algebras and the Monster, Pure and Appl. Math., Vol. 134, Academic Press, Boston, 1988. MR996026|(90h:17026)

[Hö95] G. Höhn, Selbstduale Vertexoperatorsuperalgebren und das Babymonster, dissertation, Rheinische Friedrich-Wilhelms-Universität Bonn, Bonn, 1995.

[Hö03a] G. Höhn, Self-dual codes over the Kleinian four group, Math. Ann. 327 (2003), 227-255. MR2015068 (2004i:94063)

[Hö03b] G. Höhn, Genera of vertex operator algebras and three-dimensional topological quantum field theories, Fields Inst. Commun. 39 (2003), 89-107. MR2029792 (2005m:11077)

[Hö08] G. Höhn, Conformal designs based on vertex operator algebras, Adv. Math. 217 (2008), 2301-2335. MR2388095 (2009e:17055)

[KKM91] M. Kitazume, T. Kondo and I. Miyamoto, Even lattices and doubly-even codes, J. Math. Soc. Japan 43 (1991), 67-87. MR1082423 (92b:11041) 
[Sh04] H. Shimakura, The automorphism group of the vertex operator algebra $V_{L}^{+}$for an even lattice $L$ without roots, J. Algebra 280 (2004), 29-57. MR2081920 (2005d:17038)

[Sh06] H. Shimakura, The automorphism groups of the vertex operator algebras $V_{L}^{+}$: general case, Math. Z. 252 (2006), 849-862. MR2206630 (2007d:17040)

[Sh07] H. Shimakura, Lifts of automorphisms of vertex operator algebras in simple current extensions, Math. Z. 256 (2007), 491-508. MR2299567(2008f:17044)

[Sh11] H. Shimakura, An $E_{8}$-approach to the moonshine vertex operator algebra, J. London Math. Soc. 83 (2011), 493-516.

[Ve79] B.B. Venkov, Odd unimodular lattices, algebraic numbers and finite groups, Zap. Naučn. Sem. Leningrad. Otdel. Mat. Inst. Steklov 86 (1979), 40-48. MR535479 (81a:10043)

[Wi41] E. Witt, Eine Identität zwischen Modulformen zweiten Grades, Abh. Math. Sem. Hansischen Univ. 14 (1941), 323-337. MR0005508 (3:163d)

Department of Mathematics, Aichi University of Education, 1 Hirosawa, Igaya-cho, KARIYA-CITY, Aichi 448-8542, JAPAN

E-mail address: shima@auecc.aichi-edu.ac.jp 